\title{
LEVANTAMENTO DE FUNGOS EM SEMENTES DE TRIGO${ }^{1}$
}

\author{
Leimi Kobayasti ${ }^{2}$, Aline Pellozo Pires ${ }^{2}$
}

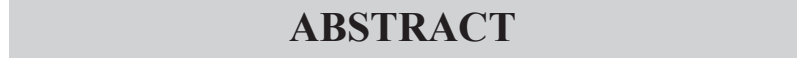

\section{SURVEY OF FUNGI IN WHEAT SEEDS}

The occurrence of pathogenic fungi in wheat seeds has been frequently reported, and this association is sometimes responsible for decreasing seeds physiologic quality, as well as introducing and disseminating pathogens and transferring early pathogens to the progeny. The objective of this study was to perform the first survey of fungi associated with upland and irrigated wheat seeds, in the Mato Grosso State, Brazil. The Brilhante, BRS 264, Aliança, BR 18, BRS 254, and IAC 350 cultivars were used. The health analysis was performed with the aid of the Blotter test adapted to water restriction $(\mathrm{NaCl}-1.0 \mathrm{MPa})$, with eight replications (25 seeds per plate), totalizing 200 seeds per sample. The fungi identification was based on their morphologic features and their incidence was quantified. The results showed variability in diversity and incidence, with a total of 20 fungi genera in the 32 samples. Cladosporium cladosporioides and Bipolaris sorokiniana were present in $96.87 \%$ of the samples, followed by Fusarium graminearum, with $75.00 \%$, and Pyricularia grisea, with $59.37 \%$.

KEY-WORDS: Triticum aestivum L.; water restriction; phytopathogenic fungi.

\section{INTRODUÇÃO}

Cerca de $90 \%$ da produção nacional de trigo está concentrada no Sul do Brasil. O cereal vem sendo introduzido paulatinamente na região do Cerrado, sob irrigação ou sequeiro (Embrapa 2007).

Desde dezembro de 2003, o Estado de Mato Grosso está oficialmente incluso no zoneamento agrícola do Ministério daAgricultura, Pecuária eAbastecimento (MAPA), por meio da Portaria 131, a qual estabelece o plantio de trigo sequeiro entre $1^{\circ}$ e 29 de fevereiro, em regiões com altitude igual ou superior a $750 \mathrm{~m}$, e o plantio irrigado entre 21 de abril e 20 de maio, em municípios com altitude superior a $600 \mathrm{~m}$ (Brasil 2003).

\section{RESUMO}

A presença de fungos patogênicos em sementes de trigo tem sido relatada com frequência, e esta associação é responsável, muitas vezes, pela redução da qualidade fisiológica das sementes, introdução e disseminação de patógenos e transmissão precoce de patógenos à progênie. Assim, objetivouse, neste trabalho, efetuar o primeiro levantamento de fungos associados a sementes de trigo irrigado e sob sistema de sequeiro, em Mato Grosso. As cultivares utilizadas foram Brilhante, BRS 264, Aliança, BR 18, BRS 254 e IAC 350. A avaliação da sanidade foi feita pelo método de incubação em papel filtro (Blotter test), modificado com restrição hídrica $(\mathrm{NaCl}-1,0 \mathrm{MPa})$, com oito repetições (25 sementes por placa), totalizando 200 sementes por amostra. A identificação dos fungos foi feita com base em suas características morfológicas, e suas incidências foram quantificadas. Os resultados apresentaram variações na diversidade e incidência, com a presença de 20 gêneros de fungos, nas 32 amostras analisadas. Os fungos Cladosporium cladosporioides e Bipolaris sorokiniana foram encontrados em 96,87\% das amostras analisadas, Fusarium graminearum em 75,00\% e Pyricularia grisea em 59,37\%.

PALAVRAS-CHAVE: Triticum aestivum L.; restrição hídrica; fungos fitopatogênicos.

A cultura do trigo apresenta excelente potencial de expansão, na região do Cerrado, boa produtividade e qualidade industrial, além de ser uma alternativa para a rotação de culturas. Estas características auxiliam no processo de implantação, incentivo e manutenção da cultura nesta região.

Patógenos transportados por sementes podem associar-se às mesmas de diferentes maneiras, contaminando-as, superficialmente, ou colonizando os seus tecidos internos. A contaminação superficial da semente por um fungo está mais sujeita à ação de alguns fatores externos, que podem reduzir as chances de sua transmissão à progênie, como, por exemplo, o tratamento com

1. Trabalho recebido em nov./2010 e aceito para publicação em nov./2011 (nº registro: PAT 12388/ DOI: 10.5216/pat.v41i4.12388).

2. Universidade Federal de Mato Grosso, Faculdade de Agronomia, Medicina Veterinária e Zootecnia, Departamento de Fitotecnia e Fitossanidade, Cuiabá, MT, Brasil.E-mails: leimi@cpd.ufmt.br, apellozo@gmail.com. 
agentes desinfestantes e a presença de antagonistas (Teixeira et al. 1997).

Segundo Machado (1988), dentre os agentes patogênicos, os fungos são os mais ativos, apresentando maior habilidade em penetrar diretamente nos tecidos vegetais e lá alojarem-se mais facilmente. $\mathrm{O}$ inóculo fúngico pode ser transportado via semente, na forma de micélio e/ou de esporos, mas a taxa de transmissão do patógeno, dentre outros fatores, depende, fundamentalmente, da quantidade e localização do inóculo na semente (Neergaard 1979, Tanaka \& Machado 1985).

A presença de fungos patogênicos em sementes de trigo tem sido relatada com frequência, em diversos países, inclusive no Brasil (Neergaard 1979, Richardson 1979 e 1981, Diehl et al. 1985, Igarashi 1988, Lasca et al. 1988, Goulart \& Paiva 1992 e 1993). Estes autores evidenciaram a presença de vários gêneros, destacando patógenos como Pyricularia grisea (Cooke) Sacc., Bipolaris sorokiniana (Sacc. in Sorok.) Schoem., Drechslera tritici-repentis (Died) Shoem., Stagonospora nodorum (Berk.) Castellani \& Germano, Septoria tritici (Rob. ex. Desm.) e Giberella zeae (Schw.) Petch, que causam sérios danos e limitam a produtividade, no Brasil.

De modo geral, vários danos podem ser provocados por patógenos associados a sementes, dentre eles a morte em pré-emergência, podridão radicular, tombamento de mudas, manchas necróticas em folhas e caules, deformações, como hipertrofias e subdesenvolvimento, descoloração de tecidos e infecções latentes (Neergaard 1979).

Diante disto, este trabalho foi realizado com o objetivo de se efetuar o primeiro levantamento de fungos associados a sementes de trigo, nos sistemas sequeiro e irrigado, em Mato Grosso, na safra 2007.

\section{MATERIAL E MÉTODOS}

O trabalho foi realizado no Laboratório de Fitopatologia da Faculdade de Agronomia e Medicina Veterinária da Universidade Federal de Mato Grosso, Campus de Cuiabá, no período de agosto a novembro de 2007.

Foram realizadas análises sanitárias das sementes de trigo, antes da semeadura, cujas cultivares foram Brilhante, BRS 264, Aliança, BR 18 e IAC 350, as quais foram doadas pela Empresa Matogrossense de Pesquisa, Assistência e Extensão Rural
S/A, Cuiabá (MT). Após o cultivo, desenvolvimento e a colheita destas cultivares, em todo o Estado, suas sementes, mais as da BRS 254, foram analisadas. Para trigo de sequeiro, as sementes foram provenientes dos municípios de Jaciara, Campo Verde, Alto Garças e Alto Taquari, e, para sistema irrigado, de Lucas do Rio Verde, Campo Verde, Primavera do Leste e Sorriso.

A análise de sanidade das sementes de trigo foi realizada pelo método de incubação em papel filtro, modificado com restrição hídrica, utilizando-se, como soluto, $\mathrm{NaCl}$ - 1,0 MPa (Farias et al. 2003). Como substrato, foram utilizadas três folhas de papel filtro esterilizadas e umedecidas com solução de restrição hídrica e acondicionadas em placas de Petri de $15 \mathrm{~cm}$ de diâmetro. As sementes foram distribuídas, uniformemente, sobre o substrato de papel, em oito repetições, representadas por uma placa com 25 sementes, totalizando 200 sementes por amostra. Em seguida, foram incubadas sob temperatura de $20^{\circ} \mathrm{C} \pm 2^{\circ} \mathrm{C}$ e mantidas, por sete dias, sob regime alternado de 12 horas de luz. Após este período, foi feita a identificação dos fungos, com base em suas características morfológicas (Barnett \& Hunter 1998, Alexopoulos et al. 1996, Leslie \& Summerell 2006) e quantificada a incidência.

\section{RESULTADOS E DISCUSSÃO}

Os patógenos encontrados nas sementes apresentaram variações na diversidade e incidência. Foram observados 20 gêneros de fungos, nas 32 amostras analisadas, incluindo as da semeadura. Foi observada a presença de Cladosporium cladosporioides e Bipolaris sorokiniana em 96,87\% das amostras analisadas, Fusarium graminearum em $75 \%$ e Pyricularia grisea em 59,37\% (Tabelas 1 a 4).

Ainda foram encontrados, associados às sementes de trigo, os fungos Colletotrichum sp., Phomopsis sp., Eurotium sp., Phoma sp., Curvularia sp., Rhizopus stolonifer, Cercospora sp., Epicoccum sp., Trichothecium sp., Mucor sp., Periconia sp. e Absidia sp., apenas em algumas cultivares, os quais foram suprimidos das tabelas, em função de sua baixa incidência.

A presença de fungos como Rhizoctonia solani (Tabelas 2 a 4), Alternaria alternata (Tabelas 1 a 4), Phoma sp., Cercospora sp. e Phomopsis sp. evidencia o transporte e a associação de patógenos causadores de doenças, em diversas culturas, cau- 
Tabela 1. Percentagem de incidência de fungos, em sementes de trigo utilizadas na semeadura da safra 2007, no Estado de Mato Grosso (Cuiabá, MT, 2007).

\begin{tabular}{lrrrrr}
\hline \multirow{2}{*}{ Patógenos } & \multicolumn{4}{c}{ Cultivares } \\
\cline { 2 - 5 } & Brilhante & BR 264 & Aliança & BR 18 & IAC 350 \\
\cline { 2 - 5 } & \multicolumn{5}{c}{ Incidência de fungos (\%) } \\
\cline { 2 - 6 } & 15,0 & 27,5 & 16,8 & 26,0 & 30,0 \\
Aspergillus spp. & 8,0 & 27,5 & 27,0 & 24,0 & 30,0 \\
Penicillium spp. & 0,5 & 0,0 & 0,0 & 0,0 & 0,0 \\
Fusarium sp. & 2,0 & 2,5 & 4,0 & 4,0 & 0,0 \\
Bipolaris sorokiniana. & 0,5 & 0,0 & 2,0 & 1,5 & 0,0 \\
Curvularia lunata & 3,0 & 0,0 & 0,0 & 0,0 & 0,0 \\
Alternaria alternata & 15,5 & 19,5 & 0,5 & 5,0 & 0,0 \\
Cladosporium cladosporioides & 0,0 & 9,5 & 0,0 & 10,0 & 0,0 \\
Fusarium verticillioides & 0,0 & 1,0 & 0,0 & 1,5 & 0,0 \\
Fusarium graminearum & & & & & \\
\hline
\end{tabular}

Tabela 2. Percentagem de incidência de fungos, em sementes de trigo cultivadas em sequeiro, nos municípios de Jaciara, Campo Verde, Alto Taquari e Alto Garças (Cuiabá, MT, 2007).

\begin{tabular}{|c|c|c|c|c|c|c|c|c|c|c|}
\hline \multirow{4}{*}{ Patógenos } & \multicolumn{3}{|c|}{ - Jaciara } & \multirow[t]{2}{*}{$\begin{array}{c}\text { Campo } \\
\text { Verde }\end{array}$} & \multicolumn{4}{|c|}{ - Alto Taquari - } & \multicolumn{2}{|c|}{ Alto Garças } \\
\hline & \multicolumn{9}{|c|}{ Cultivares } & \\
\hline & Brilhante & Aliança & BR 18 & BR 18 & Brilhante & Aliança & BR 18 & IAC 350 & Brilhante & BR 18 \\
\hline & \multicolumn{10}{|c|}{ Incidência de fungos (\%) } \\
\hline Pyricularia grisea & 43,0 & 63,0 & 0,0 & 0,0 & 24,0 & 45,0 & 33,5 & 36,0 & 38,0 & 33,5 \\
\hline Bipolaris sorokiniana & 8,0 & 13,5 & 5,0 & 22,5 & 10,5 & 7,0 & 4,5 & 21,0 & 8,5 & 4,5 \\
\hline Alternaria alternata & 3,5 & 2,0 & 4,0 & 0,0 & 0,0 & 4,5 & 2,0 & 4,5 & 6,0 & 0,0 \\
\hline Cladosporium cladosporioides & 40,0 & 20,0 & 46,5 & 33,0 & 27,0 & 37,5 & 39,0 & 42,5 & 38,0 & 30,5 \\
\hline Rhizoctonia solani & 3,0 & 0,0 & 0,0 & 16,0 & 0,0 & 0,0 & 0,0 & 0,0 & 0,0 & 0,0 \\
\hline Fusarium verticillioides & 26,0 & 20,5 & 10,0 & 0,0 & 6,5 & 9,0 & 11,5 & 11,0 & 19,0 & 13,5 \\
\hline Fusarium graminearum & 6,5 & 2,5 & 0,0 & 0,0 & 13,5 & 4,5 & 3,5 & 11,0 & 6,0 & 7,5 \\
\hline Fusarium pallidoroseum & 3,0 & 2,0 & 6,0 & 0,0 & 2,5 & 7,5 & 4,5 & 5,5 & 1,5 & 7,5 \\
\hline Nigrospora sp. & 12,0 & 25,5 & 9,0 & 0,0 & 6,0 & 8,5 & 7,0 & 14,5 & 8,5 & 13,5 \\
\hline Aspergillus spp. & 0,0 & 0,0 & 0,0 & 0,0 & 0,0 & 0,0 & 0,0 & 0,0 & 0,0 & 3,5 \\
\hline
\end{tabular}

Tabela 3. Percentagem de incidência de fungos, em sementes de trigo cultivadas sob irrigação, nos municípios de Campo Verde e Primavera do Leste (Cuiabá, MT, 2007).

\begin{tabular}{|c|c|c|c|c|c|c|c|c|c|c|c|}
\hline \multirow{4}{*}{ Patógenos } & \multicolumn{5}{|c|}{ Campo Verde } & \multicolumn{6}{|c|}{ Primavera do Leste } \\
\hline & \multicolumn{11}{|c|}{ Cultivares } \\
\hline & \multicolumn{11}{|c|}{ Brilhante BR 18 IAC 350 Aliança BRS 264 Brilhante BR 18 IAC 350 Aliança BRS 264 BRS 254} \\
\hline & \multicolumn{11}{|c|}{ Incidência de fungos (\%) } \\
\hline Pyricularia grisea & 3,5 & 39,5 & 3,0 & 5,5 & 0,0 & 7,0 & 0,0 & 0,0 & 36,5 & 3,5 & 21,5 \\
\hline Bipolaris sorokiniana & 7,5 & 1,0 & 4,5 & 7,5 & 6,0 & 8,5 & 5,5 & 6,0 & 4,5 & 0,5 & 0,5 \\
\hline Alternaria alternata & 18,0 & 2,5 & 5,0 & 16,0 & 2,5 & 1,5 & 7,5 & 3,5 & 1,0 & 0,5 & 3,0 \\
\hline Cladosporium cladosporioides & 47,5 & 37,5 & 55,0 & 70,5 & 63,5 & 12,5 & 3,0 & 26,0 & 73,5 & 50,0 & 78,0 \\
\hline Fusarium verticillioides & 9,5 & 4,5 & 7,0 & 18,0 & 9,0 & 6,5 & 36,0 & 1,0 & 2,5 & 22,5 & 8,0 \\
\hline Fusarium graminearum & 5,0 & 1,5 & 10,5 & 9,0 & 2,0 & 6,5 & 6,0 & 2,0 & 1,0 & 0,0 & 0,0 \\
\hline Fusarium pallidoroseum & 10,5 & 3,0 & 9,5 & 2,0 & 1,5 & 1,0 & 11,5 & 1,5 & 0,0 & 0,0 & 0,0 \\
\hline Nigrospora $\mathrm{sp}$ & 3,5 & 3,5 & 7,5 & 9,0 & 10,0 & 29,0 & 3,0 & 6,0 & 1,5 & 2,0 & 3,0 \\
\hline Rhizoctonia solani & 7,0 & 4,5 & 0,0 & 0,0 & 0,0 & 0,0 & 3,0 & 0,0 & 0,0 & 0,0 & 0,0 \\
\hline Penicillium spp. & 33,0 & 5,5 & 33,5 & 38,5 & 0,5 & 35,5 & 1,5 & 55,0 & 0,0 & 0,0 & 0,0 \\
\hline Aspergillus spp. & 21,5 & 0,0 & 15,0 & 14,0 & 5,0 & 16,5 & 0,0 & 6,5 & 0,0 & 0,0 & 0,0 \\
\hline
\end{tabular}

sando prejuízos econômicos, seja em grãos ou sementes. Os demais fungos encontrados nas análises de sementes, como Trichothecium sp., Curvularia sp., Nigrospora sp., Epicoccum sp., Periconia sp., Rhizopus stolonifer, Absidia sp. e Mucor sp., são de importância secundária, em sementes de trigo, e fazem parte do complexo de patógenos causadores de manchas em grãos.

Alternaria alternata, Curvularia lunata, Epicoccum purpurascens, F. verticillioides e outras 
Tabela 4. Percentagem de incidência de fungos, em sementes de trigo cultivadas sob irrigação, nos municípios de Lucas do Rio Verde e Sorriso (Cuiabá, MT, 2007).

\begin{tabular}{|c|c|c|c|c|c|c|}
\hline \multirow{4}{*}{ Patógenos } & - & do Rio & - & & Sorriso - & t \\
\hline & \multicolumn{6}{|c|}{ Cultivares } \\
\hline & BR 18 & BR 264 & IAC 350 & BR 18 & BRS 264 & BRS 254 \\
\hline & \multicolumn{6}{|c|}{ Incidência de fungos (\%) } \\
\hline Pyricularia grisea & 16,5 & 5,5 & 6,0 & 0,0 & 0,0 & 0,0 \\
\hline Bipolaris sorokiniana & 7,0 & 4,0 & 7,5 & 14,0 & 26,5 & 21,5 \\
\hline Alternaria alternata & 2,0 & 0,5 & 1,0 & 0,0 & 17,5 & 6,0 \\
\hline Cladosporium. Cladosporioides & 33,5 & 9,0 & 9,5 & 5,5 & 18,5 & 23,0 \\
\hline Fusarium verticillioides & 7,0 & 4,5 & 8,0 & 53,0 & 35,5 & 39,5 \\
\hline Fusarium graminearum & 8,0 & 10,5 & 13,5 & 0,0 & 13,0 & 10,5 \\
\hline Fusarium pallidoroseum & 8,0 & 4,5 & 2,0 & 35,0 & 12,0 & 20,5 \\
\hline Nigrospora sp. & 1,0 & 4,5 & 2,5 & 5,5 & 7,0 & 7,0 \\
\hline Rhizoctonia solani & 0,0 & 0,0 & 0,0 & 0,0 & 17,0 & 16,0 \\
\hline Aspergillus spp. & 0,0 & 0,0 & 0,0 & 0,5 & 0,0 & 0,0 \\
\hline
\end{tabular}

espécies de Fusarium foram relatadas, em associação a sementes de trigo, por Malaker et al. (2008). Além destes fungos, Fakhrunnisa \& Ghaffar (2006) também relataram, associados ao trigo, Absidia sp., Nigrospora oryzae, Rhizoctonia solani, Rhizopus sp. e Trichothecium roseum. Brancão et al. (2002) destacaram, ainda, a presença de Phoma sp. Já Goulart \& Paiva (1992), além dos fungos acima citados, observaram a presença de Mucor sp. e Phomopsis sp.

A incidência do fungo Cladosporium cladosporioides, observado em todas as amostras de sementes analisadas, variou de $0 \%$ a $78 \%$ (Tabelas 1 a 4). Este fungo é patogênico a muitas espécies de plantas, podendo causar redução na germinação de sementes de trigo (Dorovskaya \& Khasanava 1974 apud Rajput et al. 2005), e, também, tem sido relatado em associação a sementes de trigo (Goulart \& Paiva 1992, Brancão et al. 2002, Dorovskaya \& Khasanava 1974 apud Rajput et al. 2005, Fakhrunnisa \& Ghaffar 2006, Duan et al. 2007, Malaker at al. 2008). Além disto, Garcia Júnior et al. (2008) relataram que a detecção de $F$. graminearum pode ser prejudicada pela alta incidência de fungos saprofíticos, como Cladosporium sp.

Nas análises realizadas para as sementes utilizadas na semeadura (Tabela 1), foram observados patógenos como Bipolaris sorokiniana e $F$. graminearum, com percentagens máximas de 4,0\% e 1,5\%, respectivamente. Fungos como Aspergillus spp. e Penicillium spp. foram encontrados em todas as cultivares plantadas, com percentagens variando de $15 \%$ a $30 \%$ e de $8 \%$ a $30 \%$, respectivamente.

As sementes do sistema trigo de sequeiro (Tabela 2), provenientes dos municípios de Jaciara, Campo Verde, Alto Garças e Alto Taquari, apresentaram variações na incidência de $P$. grisea. O patógeno foi observado nas cultivares Aliança e Brilhante, em Jaciara (63\% e 43\%, respectivamente). Já em Alto Taquari, as cultivares Aliança, IAC 350, BR 18 e Brilhante apresentaram 45,0\%,36,0\%, 33,5\% e $24,0 \%$ de incidência do fungo, respectivamente. Em Alto Garças, a incidência, nas sementes das cultivares Brilhante e BR 18, foi de 38,0\% e 33,5\%, respectivamente. Estes índices são considerados altos, em função da importância da doença e do grau de prejuízo que o patógeno pode causar à cultura.

Para $B$. sorokiniana, nos quatro municípios em que as sementes foram plantadas em sistema de sequeiro, houve variação de 4,5\% (BR 18, em Alto Taquari e Alto Garças) a 22,5\% (BR 18, em Campo Verde), enquanto, para $F$. graminearum, as percentagens variaram de $0 \%$ (BR 18, em Jaciara e Campo Verde) a 13,5\% (Brilhante, em Alto Taquari) (Tabela 2).

Nas sementes de trigo provenientes de sistema irrigado, houve maior diversidade de gêneros de fungos, quando comparados ao sistema de sequeiro (Tabelas 3 e 4). P. grisea pôde ser observado em todos os locais onde se plantou trigo irrigado, exceto no município de Sorriso. Em Campo Verde, a presença do fungo variou de 3,0\% (IAC 350) a 39,5\% (BR 18). Para B. sorokiniana, a variação foi de $0,5 \%$ (BRS 264 e BRS 254, em Primavera do Leste) a 26,5\% (BRS 264, em Sorriso). O fungo F. graminearum variou de $0 \%$ (BRS 264 e BRS 254, em Primavera do Leste, e BR 18, em Sorriso) a 13,5\% (IAC 350, em Lucas do Rio Verde).

A brusone do trigo, causada por P. grisea, é uma das doenças mais preocupantes da parte aérea. A importância desta doença decorre das reduções no rendimento e na qualidade de grãos, sendo que as perdas podem atingir até 50\% (Reis \& Casa 2005). 
Quando a infecção é precoce (início das fases de florescimento e enchimento de grão), os grãos, se houver, apresentam-se deformados, pequenos e com baixo peso específico, e a maioria é eliminada nos processos de colheita e beneficiamento (Embrapa 2004).

É importante observar que não houve identificação de $P$. grisea na amostra semeada, porém, a sua presença, em até $63,0 \%$ (Tabela 2 ), na cultivar Aliança, em Jaciara, nas sementes colhidas, é preocupante para esta região do Estado de Mato Grosso, onde as condições são propícias ao desenvolvimento de brusone, principalmente no sistema de sequeiro. Vale ressaltar que as sementes contaminadas são importante fonte de inóculo primário da doença, em novas áreas (Goulart \& Paiva 1990, Urashima et al. 1999), e que existe correlação direta entre a incidência de brusone em espigas de trigo, no campo, e a percentagem de sementes colhidas com a presença do patógeno (Goulart et al. 1995).

A mancha foliar, ou helmintosporiose, causada por B. sorokiniana, é considerada uma das doenças mais danosas à cultura do trigo, chegando a acarretar perdas de $20-80 \%$, no rendimento deste cereal. Sob condições muito favoráveis, a doença ocorre durante todo o ciclo da cultura (Barros et al. 2006). A semente infectada por este patógeno constitui-se no principal mecanismo de sobrevivência do fungo, entre safras agrícolas, o que garante a continuidade do seu ciclo de vida. Ainda, pode ter influência negativa direta na germinação e no estabelecimento de pastagens. Já em sementes armazenadas, tem a capacidade de sobreviver como micélio, no endosperma da semente, sob condições de estresse hídrico, e colonizar o sistema radicular e a parte aérea, durante a germinação (Neergaard 1979).

Por meio de sementes infectadas, fungos como B. sorokiniana são introduzidos em áreas novas de cultivo, ou em lavouras sob rotação de culturas, sendo, posteriormente, transmitidos para os órgãos radiculares (mesocótilo e raízes seminais) e aéreos (coleóptilo e plúmula). Para o trigo, estudos sobre a eficiência de transmissão deste fungo relatam valores de até $88 \%$ ao coleóptilo (Reis \& Forcelini 1993), $68 \%$ ao mesocótilo (Forcelini 1992) e 38\% à plúmula (Toledo et al. 1996). Por causa da elevada transmissão que $B$. sorokiniana apresenta, Reis (1987) considera que o controle deste fungo deve ser orientado à sua erradicação na semente, a fim de se reduzir o inóculo primário, na lavoura. A incidência deste fungo, em
$96,87 \%$ das amostras analisadas, o evidencia como patógeno importante, associado a sementes de trigo. Estes resultados concordam com os obtidos por Goulart \& Paiva (1992 e 1993) e Diehl et al. (1985).

F. graminearum (teleomorfo Giberella zeae), causador da giberela, presente em $75 \%$ das amostras analisadas (Tabelas 1 a 4), é predominante em áreas onde se plantam arroz e milho, além de ser favorecido pela umidade contínua (mínimo de 24 horas) e temperaturas de $20-30^{\circ} \mathrm{C}$ (Diekmann \& Putter 1995). As infecções causadas por este patógeno podem afetar tanto aspectos físicos quanto fisiológicos da semente, incluindo o seu tamanho, peso, composição e qualidade (Bechtel et al. 1985), permitindo que ocorram severas perdas no rendimento, as quais podem ser superiores a $50 \%$, além de afetar a qualidade dos grãos susceptíveis (Snijders 1990).

Garcia Júnior (2006) evidenciou a transmissão de $F$. graminearum a diferentes partes da plântula de trigo, a partir de sementes naturalmente infectadas pelo patógeno. A utilização de sementes portadoras deste patógeno permite a sua reintrodução em áreas onde o fungo irá se estabelecer na haste, além de colonizar folhas senescentes, de modo saprofítico, culminando no aumento da população deste patógeno, justamente no maior período de susceptibilidade da planta, que é durante a formação das espiguetas (Bergstron 1993).

A semente de trigo é o principal e mais eficiente veículo de transmissão e disseminação de patógenos, bem como meio de sobrevivência dos mesmos, em contato direto com o hospedeiro (Machado 1982). Isto implica na introdução de patógenos em áreas ainda livres deles e de raças mais virulentas, ainda não existentes, bem como na ocorrência de infecção, nos estádios iniciais de desenvolvimento da planta (Tanaka \& Machado 1985). Além disto, a frequente introdução de patógenos pelas sementes tende a aumentar a incidência de doenças já existentes em uma área. Muitas doenças que ocorrem no Brasil foram introduzidas por meio de sementes que carregavam, interna ou externamente, organismos patogênicos (Tanaka 1982).

Com a cultura do trigo sendo introduzida no Estado de Mato Grosso, é imprescindível a recomendação de análise fitossanitária e tratamento de sementes, como uma das medidas de controle para se potencializar a sua produtividade. Desta forma, a adoção da prática de tratamento de sementes, bem como dos produtos a serem utilizados, deve ser 
decidida preventivamente ao surgimento da doença e associada a outras técnicas que assegurem um potencial elevado de rendimento à lavoura.

\section{CONCLUSÃO}

Dentre as cultivares avaliadas em Mato Grosso, foram observados 20 gêneros de fungos, sendo Bipolaris sorokiniana, Fusarium graminearum e Pyricularia grisea os de maior importância para a cultura do trigo.

\section{AGRADECIMENTOS}

Ao Sr. Hortêncio Paro (EMPAER-MT), pelas amostras de sementes de trigo.

\section{REFERÊNCIAS}

ALEXOPOULOS, C. J.; MIMS, C. W.; BLACKWELL, M. Introductory mycology. 4. ed. New York: John Wiley \& Sons, 1996.

BARNETT, H. L.; HUNTER, B. B. Illustrated genera of imperfect fungi. 4. ed. Saint Paul: The American Phytopathological Society, 1998.

BARros, B. C.; CASTRO, J. L.; PATRÍCIO, F. R. A. Resposta de cultivares de trigo (Triticum aestivum L.) ao controle químico das principais doenças fúngicas da cultura. Summa Phytopathologica, Botucatu, v. 32, n. 3, 2006. Disponível em: <http://www.scielo.br/scielo. php? pid $=$ S0100-54052006000300005\&script $=$ sci arttext>. Acesso em: 17 nov. 2007.

BECHTEL, D. B. et al. The effects of Fusarium graminearum infection on wheat kernels. Cereal Chemistry, Saint Paul, v. 62, n. 2, p. 191-197, 1985.

BERGSTRON, G. C. Scab (head blight). In: MATHUR, S. B.; CUNFER, B. M. (Eds.). Seed borne diseases and seed health testing of wheat. Copenhagen: Hellerup, 1993. p. 83-93.

BRANCÃO, N. et al. Ocorrência de fungos em sementes de sorgo, milho, soja e trigo. 2002. Disponível em: $<$ http://www.cpact.embrapa.br/publicacoes/download/ comunicados/comunicado76.pdf $>$. Acesso em: 07 jun. 2011.

BRASIL. Ministério da Agricultura, Pecuária e Abastecimento (MAPA). Portaria ${ }^{\circ} 131$, de 8 de dezembro de 2003. Diário Oficial da República Federativa do Brasil, Brasília, DF, 11 dez. 2003. Disponível em: <http://extranet.
agricultura.gov.br/sislegis/action/detalhaAto.do?method= gravarAtoPDF \& tipo $=$ POR \&numeroAto $=00000131 \&$ seq Ato $=000 \&$ valorAno $=2003 \&$ orgao $=$ CER $/$ MAPA\&codTip $\mathrm{o}=\&$ desItem $=\&$ desItemFim $>$. Acesso em: 15 dez. 2011.

DIEHL, J. A.; BACALTCHUK, B.; FERREIRA FILHO, A. Fungos patogênicos presentes em sementes de trigo no Rio Grande do Sul e Paraná, 1984. Revista Brasileira de Sementes, Brasília, DF, v. 1, n. 1, p. 81-89, 1985.

DIEKMANN, M.; PUTTER, C. A. J. Small grain temperate cereals. Roma: FAO/IPGRI, 1995.

DUAN, C. et al. Testing of seedborne fungi in wheat germplasm conserved in the National Crop Genebank of China. Agricultural Sciences in China, Beijing, v. 6, n. 6, p. 682-687, 2007.

EMPRESA BRASILEIRA DE PESQUISA AGROPECUÁRIA (Embrapa). Informações gerais sobre brusone em trigo e em cevada. Passo Fundo: Embrapa, 2004.

EMPRESA BRASILEIRA DE PESQUISA AGROPECUÁRIA (Embrapa). Trigo. 2007. Disponível em: <http://www.cnpt.embrapa.br/culturas/trigo/index. htm>. Acesso em: 27 nov. 2007.

FAKHRUNNISA, M. H. H.; GHAFFAR, A. Seed-borne mycoflora of wheat, sorghum and barley. Pakistan Journal of Botany, Karachi, v. 38, n. 1, p. 185-192, 2006.

FARIAS, C. R. J. et al. Inibição de germinação de sementes de trigo e milho em teste de sanidade em substrato de papel. Revista Brasileira de Agrociência, Pelotas, v. 9, n. 2, p. 141-144, 2003.

FORCELINI, C. A. Incidência, transmissão e controle de Bipolaris sorokiniana em sementes de trigo. 1992. 114 f. Dissertação (Mestrado em Fitopatologia)-Escola Superior de Agricultura Luiz de Queiroz, Universidade de São Paulo, Piracicaba, 1992.

GARCIA JÚNIOR, D. Fusarium graminearum em sementes de trigo (Triticum aestivum L.): detecção, efeitos e controle. 2006. 78 f. Tese (Doutorado em Fitopatologia)-Escola Superior de Agricultura Luiz de Queiroz, Universidade de São Paulo, Piracicaba, 2006.

GARCIA JÚNIOR, D.; VECHIATO, M. H.; MENTEN, J. O. M. Comparação de métodos para a detecção de Fusarium graminearum em sementes de trigo (Triticum aestivum L.). Summa Phytopathologica, Botucatu, v. 34, n. 2, p. 164-167, 2008.

GOULART, A. C. P.; PAIVA, F. de A. Fungos associados às sementes de trigo (Triticum aestivum) produzidas no Mato Grosso do Sul em 1990 e 1991. Revista Brasileira de Sementes, Brasília, DF, v. 14, n. 2, p. 221-225, 1992. 
GOULART, A. C. P.; PAIVA, F. de A. Incidência de fungos em sementes de trigo (Triticum aestivum) produzidas no Mato Grosso do Sul. Fitopatologia Brasileira, Brasília, DF, v. 18, n. 1, p. 107-109, 1993.

GOUlART, A. C. P.; PAIVA, F. A. Transmissão de Pyricularia grisea através de sementes de trigo (Triticum aestivum). Fitopatologia Brasileira, Brasília, DF, v. 15, n. 4, p. 359-362, 1990.

GOULART, A. C. P., PAIVA, F. A.; ANDRADE, P. J. M. Relação entre incidência da brusone do trigo e a presença de Pyricularia grisea nas sementes colhidas. Fitopatologia Brasileira, Brasília, DF, v. 20, n. 2, p. 184-189, 1995.

IGARASHI, S. Uma análise da ocorrência de "brusone" do trigo no Paraná. In: SEMINÁRIO SOBRE MELHORAMENTO PARA RESISTÊNCIA DE TRIGO A ENFERMIDADES, 1., 1988, Passo Fundo. Resumos... Passo Fundo: Embrapa Trigo, 1988. Mimeografado.

LASCA, C. C.; KOHARA, E. Y.; BARROS, B. C. Incidência de Pyricularia oryzae em sementes de trigo produzidas no Estado de São Paulo. In: REUNIÃO DA COMISSÃO CENTRO-SUL BRASILEIRA DE PESQUISA DE TRIGO, 4., 1988, Campinas. Resultados de pesquisa. Campinas: Instituto Biológico, 1988. Não paginado.

LESLIE, J. F.; SUMMERELL, B. A. The Fusarium laboratory manual. Iowa: Blackwell Publishing, 2006.

MACHADO, J. C. Controle de fitopatógenos associados a sementes. Informe Agropecuário, Belo Horizonte, v. 8, n. 91 , p. 35-38, 1982.

MACHADO, J. C. Patologia de sementes: fundamentos e aplicações. Brasília, DF: MEC/ESAL/FAEPE, 1988.

MALAKER, P. K. et al. Effect of storage containers and time on seed quality of wheat. Bangladesh Journal of Agricultural Research, Bangladesh, v. 33, n. 3, p. 469477, 2008.

NEERGAARD, P. Seed pathology. 2. ed. London: Macmillan, 1979.

RAJPUT, M. A. et al. Studies on seed-borne fungi of wheat in sindh Province and their effect on seed germination. Pakistan Journal of Botany, Karachi, v. 37, n. 1, p. 181185, 2005.
REIS, E. M. Patologia de sementes de cereais de inverno. São Paulo: CNDA, 1987.

REIS, E. M.; CASA, R. T. Doenças do trigo. In: KIMATI, H. et al. (Eds.). Manual de fitopatologia. São Paulo: Agronômica Ceres, 2005. p. 631-638.

REIS, E. M.; FORCELINI, C. A. Transmissão de Bipolaris sorokiniana de sementes para órgãos radiculares e aéreos do trigo. Fitopatologia Brasileira, Brasília, DF, v. 18, n. 1, p. 76-81, 1993.

RICHARDSON, M. J. An annotated list of seed-borne diseases. 3. ed. London: CAB/CMI/STA, 1979.

RICHARDSON, M. J. Supplement I to an annotated list of seed-borne diseases. 3. ed. London: CAB/CMI/ISTA, 1981.

SNIJDERS, C. H. A. The inheritance of resistance to head blight caused by Fusarium culmorum in winter wheat. Euphytica, Dordrecht, v. 50, n. 1, p. 11-18, 1990.

TANAKA, M. A. S. Importância da utilização de sementes sadias. Informe Agropecuário, Belo Horizonte, v. 8, n. 91, p. 31-34, 1982.

TANAKA, M. A. S.; MACHADO, J. C. Patologia de sementes. Informe Agropecuário, Belo Horizonte, v. 11, n. 122, p. 40-46, 1985.

TEIXEIRA, H.; MACHADO, J. C.; VIEIRA, M. G. G. C. Influência de Colletotrichum gossypii South. no desenvolvimento inicial do algodão (Gossypium hirsutum L.) em função da localização do inóculo e desinfestação das sementes. Revista Brasileira de Sementes, Brasília, DF, v. 19, n. 1, p. 9-13, 1997.

TOLEDO, J.; ROCA, R. H.; ESCÓBAR, R. E. Transmisión, persistencia y control químico de Bipolaris sorokiniana causante de la punta negra del grano en trigo. In: CIAT. Proyecto de investigación: trigo. Santa Cruz de la Sierra: CIAT, 1996. p. 87-106.

URASHIMA, A. S. et al. Molecular analysis of the wheat blast population in Brazil with a homolog of retrotransposon MGR583. Annals of the Phytopathological Society of Japan, Tokyo, v. 65, n. 4, p. 429-436, 1999. 